\title{
Visual and Interaction Design Themes in Mobile Healthcare
}

\author{
Ben Falchuk $^{*}$
}

\begin{abstract}
The opening-up of mobile application development has enabled the launches of successful "app stores" for iPhone, Android, Blackberry and others, from which innovative mobile applications are available. The healthcare realm is not left out; scores of health and wellness-related apps are available, though most (but not all) are simplistic, highly derivative or not overly compelling from graphical, creative, or usability points of view. In this paper we take a broader look at mobile platforms, enablers, and example healthcare applications that are noteworthy or representative in one way or another - these include some of the authors own research. We look at a series of exemplary and topical real-world examples to help us identify and support several overarching themes salient to mobile healthcare services. These themes include: the use of novel interaction techniques, the use of human-computer interface to encourage and mitigate movement and activity, and the use of graphics to improve the representation and understanding of medical, wellness, and life information. This paper, then, serves as a brief survey of existing health-related applications as well as an introduction to a few thought-provoking applications that "are not here yet" but promise to have an impact on mobile healthcare.
\end{abstract}

\section{INTRODUCTION}

A growing number of medical studies now almost indisputably show the positive benefits of both physical and mental activities on overall health. Most echo the kind of insights gained by, for example, the American Heart Association:

\begin{abstract}
"...the beneficial effect of vigorous physical activity in reducing the risk of heart failure was observed .... Any amount of vigorous physical activity that caused sweating, ranging from a low of one to three times a month to a high of five to seven times a week, was associated with an 18 percent reduction in heart failure risk...." [11]
\end{abstract}

Similarly, brain-training - in which a user plays challenging quiz and problem-solving games - is of great current interest to both insurers and governments ${ }^{1}$. Both have a keen interest in understanding if brain-training (also referred to as, "continued learning ") can help to maintain mental health, memory, and concentration, thereby reducing accidents and, perhaps, the onset of mental syndromes. Meanwhile, there is evidence that getting patients communicating with positive role models may lessen the impact of a social circle of bad influences [2].

\footnotetext{
* Ben Falchuk, Ph.d., is a Senior Research Scientist at Telcordia Technologies, Piscataway, NJ 08854 USA (email: bfalchuk@research.telcordia.com)

1 "Baycrest creates Centre for Brain Fitness with \$10-million investment from Ontario Government", see http://www.baycrest.org

${ }^{2}$ http://www.globalaging.org/health/world/2008/games.htm
}

With respect to mobile wireless communications, in most nations of the world we see large or increasing penetration of mobile smartphones (i.e., those cellular phones capable of email, personal productivity applications, contact lists, and $3^{\text {rd }}$ party applications). Application stores - or "App stores" - have recently helped Apple, Blackberry, and T-Mobile to increase uptake in their smartphones by offering fun, cheap, and sometimes innovative applications for their mobile platforms.

Mobile healthcare can be thought of as the functional combination of the IT of telemedicine ${ }^{3}$ with mobile pervasive services [19]. Here, focusing largely on the big picture of the design of mobile healthcare applications, we ask, 'what are the impediments to riding mobile healthcare on top of today's hardware and software capabilities?' Usability, accessibility, security, privacy, and connectivity are several important issues. Usability - defined in ISO as the "extent to which a product can be used by specified users to achieve specified goals with effectiveness, efficiency and satisfaction in a specified context of use" - remains a growing concern; on par, we believe, with the other issues. Consider, for example, that mobility notwithstanding, a majority of legacy Web sites still suffer from poor usability ${ }^{4}$ and that developing for the mobile device [12] poses an even greater challenge ${ }^{5}$. When technical foundations and standards exist to address the above issues, focus will shift to higher level design and user-experience themes that we outline in subsequent sections of this paper.

Over the past several years, the research, intellectual property, prototypes, and pilots that we have built for our internal and external customers have given us a broad perspective in areas such as: interaction, mobile locationbased services [1][5][6], information mashups [4], mobile user experience, advanced wellness monitoring [3], and advanced visual Web and mobile applications to promote activity and discovery [7][8]. In this paper we weave lessons from our past work and relevant related work into a larger presentation of visual and interaction design themes in mobile healthcare. The remainder of this paper is structured as follows: In section two we outline some main themes that are important to mobile healthcare applications. We look at how novel computer interfaces can be used to improve healthcare applications. Then we examine how applications can motivate users to change their behavior for the better.

\footnotetext{
3 see Health Information Technology Standards Panel (HITSP)

${ }^{4}$ sites like webpagesthatsuck.com exist solely to decry design failures

5 a large fraction of mobile users who can use their cell phones to access the Internet still do not because of navigation, efficacy, availability, and other usability issues.
} 
Finally we look at how graphical interfaces can help (or hinder) the analysis and presentation of medical context and information. Finally, we conclude and summarize.

Our work is inspired by and complements the work of Consolvo et al [15] in which design (but not visual) requirements were derived from a mobile phone-based fitness program. Whereas Consolvo et al focused on more general practicalities of activity encouragement, we focus on the visual development themes. They concluded that four design requirements were key for applications that encourage physical activity: giving users credit (e.g., rewards), providing personal indicators of activity level, supporting social influence, and considering users' lifestyle factors. Elsewhere, underlying principles of mobile application design can be found in various sources, ranging from theoretical to de facto to unproven. Excellent sources at a broad level include the W3C Mobile Web Best Practices ${ }^{6}$, and the Apple iPhone Human Interface Guidelines ${ }^{7}$. In addition, decades of broad human computer interface research can be found in journals, SIG's and conferences (e.g., SIGGRAPH).

\section{THEMES}

This section identifies important themes for visual design and interaction design in mobile healthcare. This section also provides illustrative examples of each theme and describes how those examples support key healthcare scenarios.

\section{DESIGNING FOR A RANGE OF NOVEL INTERFACES}

Novel interface technologies such as multi-touch (in which a touch screen can track several touches at once) and low-cost sensors - such as pedometers - are making mobile healthcare and wellness applications exciting, viable, and tangible. Gesture-based devices, in which physical gestures are detected by a computer and converted to interface actions, have great possibility as well, for both safety (e.g., detecting patient-falls [20]) and accessibility and fun (see Wii in next section). Socalled smart-homes are, in some cases, already capable of monitoring resident whereabouts in the home as well as computing over their actions. With additional semantics and artificial intelligence, this computing includes the ability to infer activities and reason about future needs. Novel ambient interfaces will convey information to in-home patients in the best but most subtle ways (e.g., "ambient intelligence" for elderly patients [21]). Novel mass market special-purpose mobile applications are already mainstream - Apple and Nike offer an in-shoe pedometer that broadcasts steps to an iPod attachment; by counting and analyzing steps and pace the addon software can track workouts and choose music to motivate the runner. Nokia SportsTracker and Adidas MiCoach are similar but work with yet different technologies (GPS) and devices (e.g. T-mobile "Android" handsets) ${ }^{8}$. Figure 1 illustrates.

\footnotetext{
${ }^{6}$ http://www.w3.org/TR/mobile-bp

${ }^{7}$ See http://developer.apple.com/

${ }^{8}$ See: http://www.apple.com/ipod/nike, http://www.micoach.com , http://sportstracker.nokia.com
}

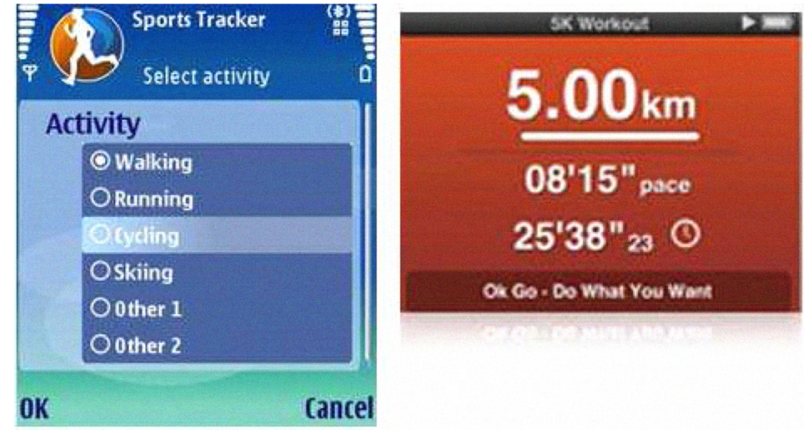

Figure 1. Interfaces for health tracking focused on sports and activities iPod/Nike (left) and Nokia Sports Tracker (right)

These activity trackers are effective and sell well because they are relatively unobtrusive (e.g., attach to one's shoe) and interwork with already-familiar and in-hand devices such as the iPod.

The Microsoft Surface touch table interface is a relatively new, but compelling platform. Though it is definitely not mobile (it's a table with a multi-touch screen and several cameras underneath), it bears mentioning for both the compelling healthcare-related applications it enables and the undeniable feeling that it will not be long before multi-touch mobile devices are capable of some of the interactions afforded by Surface. Infusion Development has built, for example, a patient consultation system for Texas Health Resources that "augments and facilitates the conversations that a doctor regularly has with his or her patients through a unique, interactive representation on the Microsoft Surface. With the use of static and active media elements, a doctor is able to demonstrate and relate complex medical procedures or conditions in layman's terms to their patients"' (see Figure 2).

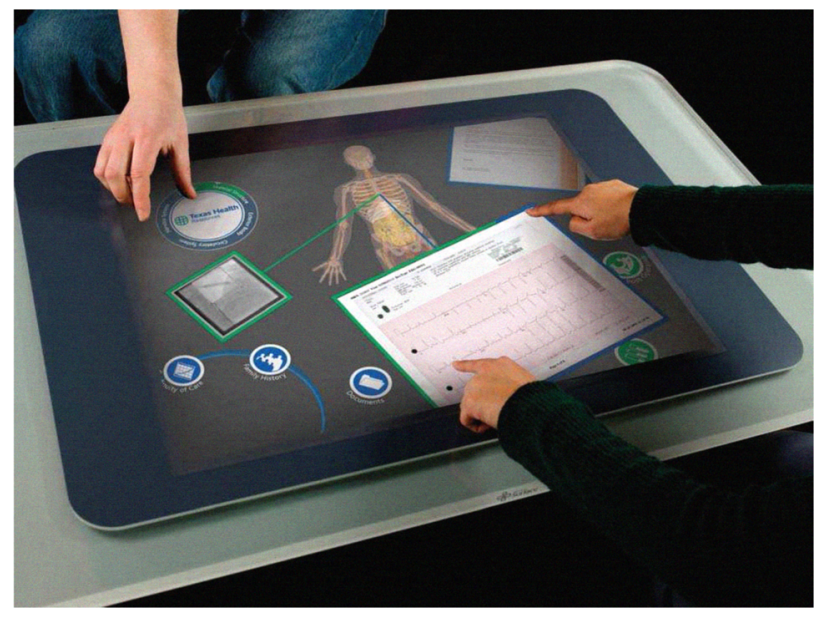

Figure 2. Texas Health Resources health records application (by Infusion Development Inc.) running on Microsoft Surface. Communications between doctors and patients are improved via multi-touches and gestures on the interactive information screen.

Elsewhere, InterKnowlogy VitruView was designed to, "help with angiography procedures in a cardiac catheter lab

\footnotetext{
${ }^{9}$ http://www.youtube.com/watch?v=Qf0WxOo304g
} 
- it is essentially a 3D multi-touch application for heart surgeries allowing health care professionals to access annotate and collaborate on patient data that persists in Microsoft HealthVault." ${ }^{10}$

Designing for a range of device interface types clearly includes designing for those devices already in user's hands. Exploiting the familiar is a real advantage (e.g., consider technologies for traumatic brain injury patients ${ }^{11}$ or elderly or dementia-afflicted patients where familiarity is essential); however, it may also mean compromising on features or refactoring existing technologies. For example, in response to the difficulty of diagnosing and treating patients in poor rural areas or areas with no doctors, UCLA's Dr. Ozcan has adapted a normal cell phone with some additional algorithms and hardware. These new add-ons - not expensive at all - together with the algorithms, allow the cell phone to become a fluid sampling machine, displaying the results of cellular-level fluids analysis. Such analysis is important in determining if, "if an HIV patient has AIDS.. or a red cell count can help determine if a patient is anemic or might have malaria" [9]. This work follws Rubinsky's UC Berkeley work in which a mobile cellphone is repurposed to fill a gap in medical imaging [10]. The World Health Organization estimates that about $3 / 4$ of the world's population has little to no access to medical imaging. Rubinsky's solution was to reuse the graphics interfaces of cellphones as displays for more heavyweight server side image analysis engines. Thus, his equipment collects imaging information, transmits it wirelessly to a central server and awaits a response. The image subsequently returned to the analyst's cellphone is displayed and analyzed by an on-site remote health professional (see Figure 3). Though resolution and screen size lack, this approach can prove more cost-effective than buying, installing and maintaining special-purpose imaging hardware in remote locations.

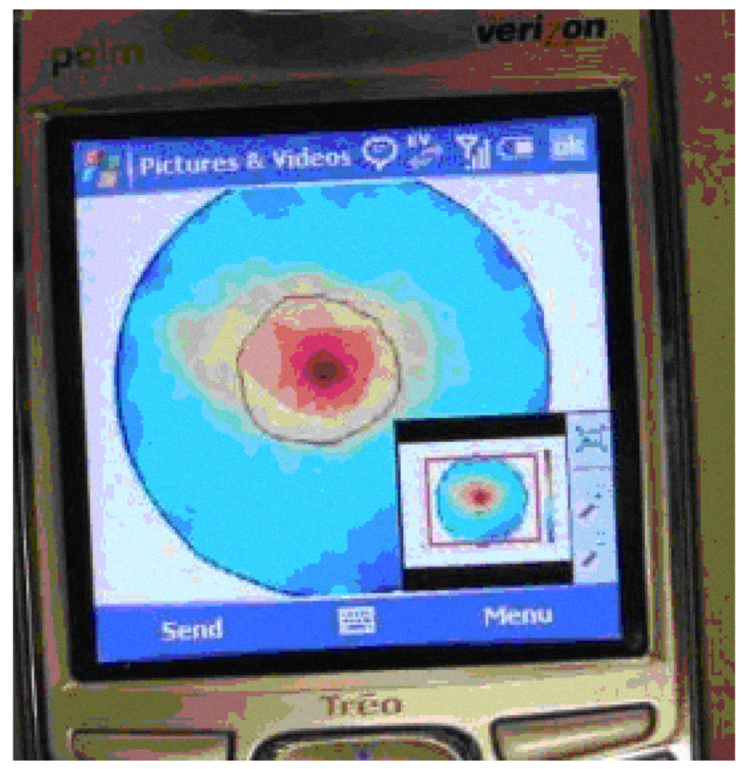

Figure 3. Cell phone technology allows data acquisition and image analysis steps to occur on different platforms.

\footnotetext{
${ }^{10} \mathrm{http} / / / \mathrm{www}$. interknowlogy.com

${ }^{11} \mathrm{http}: / /$ www.ninds.nih.gov/disorders/tbi/tbi.htm
}

Somewhat farther in the future of mobile healthcare lies the brain-computer interface (BCI). BCI is comprised of the technologies that sense brain activity and use a computer system to interpret the activities. In healthcare use cases these systems allow patients who are unable to physically move to control networked virtual and physical resources through controlled thoughts and brain activities. Other health and wellness applications of BCI are possible, though, and using a mobile computing platform is not unduly unrealistic. Several companies manufacture and sell BCI components expressly for use in video game systems ${ }^{12}$.

In summary, new and novel human interfaces combined with creative and unexpected uses of familiar technology will be of great value mobile healthcare. A diverse mix of physical screens, touch-pads, and projection devices are on the horizon. Designers of mobile healthcare need to carefully consider not only the human factors of the new interfaces [12] but also how their designs can be weaved into the dailylife of the patients using safe, familiar, or perhaps invisible (see "pervasive computing") means.

\section{DESIGNING TO ENCOURAGE AND MITIGATE MOVEMENT AND ACTIVITY}

Getting people out the door, away from their television and physically active is a major problem in the United States. The problems are compound, as inactivity leads to other bad habits; for example, "high-school students who watched more than five hours of TV each day had a higher intake of snack foods, fried foods, fast food, sugar-sweetened beverages, and trans-fats five years later" [13]. In some health models, adherence is heightened with lower perceived threats and higher perceived benefits [16]. It seems clear that these models - when applied to mobile healthcare would benefit from reward mechanisms and positive reenforcements use cases.

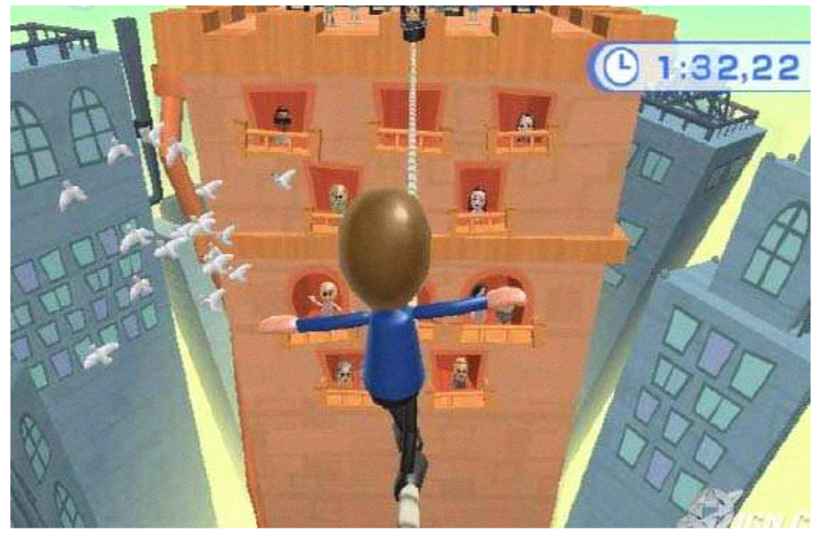

Figure 4. Wii Fit (balancing on the balance-board)

Turning these issues around, then, we pose the questions:

- How can mobile healthcare make positive activities more fun?

- How can mobile healthcare systems reward positive behaviors and provide positive reinforcements?

${ }^{12}$ E.g., see http://www.emotive.com, http://www.neurosky.com 
We have already outlined how the Nike / iPod product makes exercise routines fun by making the hassle of exercise-logging disappear. It also gives the "patient" simple motivational messages and selects music from the user's own song library to motivate the user to complete the exercise plan. The computing algorithms in this product are not great, but enormous potential exists when great computing capabilities and algorithms - such as Artificial Intelligence - are applied to reminders, encouragement and rewards [24].

The Nintendo Wii, launched around 2007, is a relatively lowcost gaming system whose principle controller is gesture-based. The controller - also equipped with buttons - uses both infrared and accelerometers to resolve its coordinates in space. In doing so, it enabled a fun new class of gaming experiences in which users holding the controller gesture in particular ways (such as "boxing" punches and tennis swings) and the in-game avatar reacts in real-time. The Wii Fit game makes use of the Wii Balance Board and puts gamers through a series of exercises, stretches and various coordination activities by requiring them to balance, step and gesture. Figure 4 shows a "tight-rope" challenge screen reacting to a gamer on the balance board. Therefore, while the majority of games before Wii required the skilled use of a controller, they allowed the gamer to remain seated. Many of the Wii's games cannot be played effectively without moving about. The promise of Wii and Wii-like systems is evident in the uptake of Wii in Senior Citizens homes where these physical activity games have become a part of physical therapy or recuperation scenarios [14]. In some cases, seniors have made these games a part of the social scene in the home and observers have noted increased camaraderie and spirits. At the moment, Wii is not a mobile system and is essentially intended to sit in the living room near the television. It is not hard, however, to imagine more portable versions of this and other related technologies that would have similar health and wellness benefits.

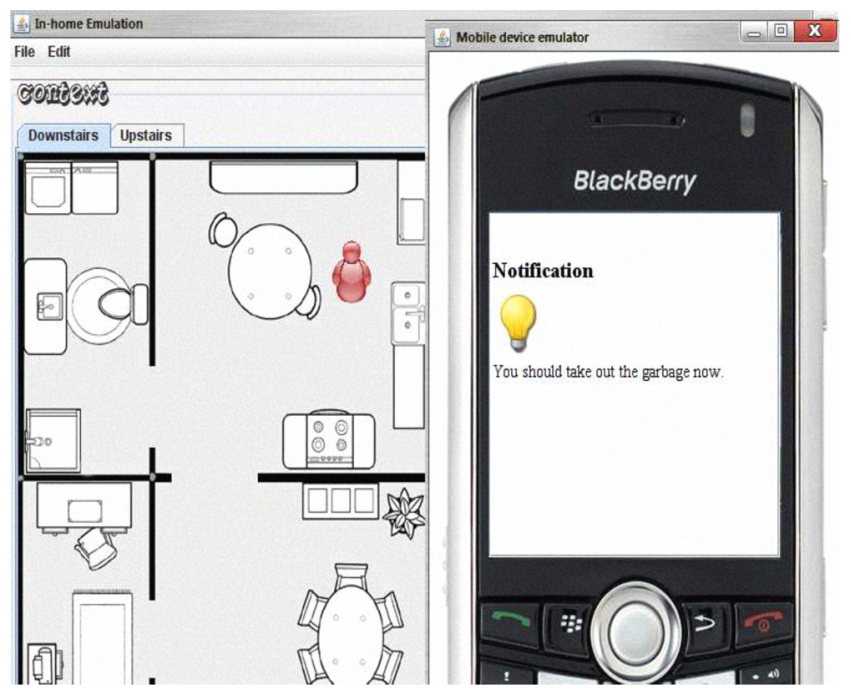

Figure 5. Indoor reminders, activity monitoring and planning prototype; the technologies of location sensing, machine inference, and workflows come together to enable compelling mobile health and wellness applications

One emerging technology dovetailing from traditional mobile Location Based Services (LBS) is micro-location services. While some such LBS systems are tailored to remind mobile users to pickup prescriptions and to help ensure compliance, others can be tailored to work in closed "micro" environments - such as offices, campuses, and so-called "smart homes" [17][24] - to provide helpful information and logging that can be shared with other household members, with clinicians, or simply used by the patient as a memoryaide. In-home health monitoring and assistance services are a growing concern ${ }^{13}$ and our research into this field has yielded some novel approaches to handling position determination and task/subtask management. Figure 5 illustrates a prototype environment which emulates a smart home equipped with room and region-based sensors (see figure left, various rooms and the emulated patient position as understood by the system). An emulated patient mobile device is shown at the right. A server-based planner runs on the patients' home computer or in the network. The goals of this work are to devise software and approaches to:

- Better locate patient location and activity within the house, taking into account the various kinds of activity and motion sensors that may be deployed, and the partial information that may be known at any time.

- Anticipate and react in real-time to patient behavior and to send them notifications that help clear up confusion, give them a task (e.g., encourage activity), or reminder them of something important.

Our prototype shows some improvements over current art techniques. We base our internal model on a Semantic modeling language - Web Ontology Language ${ }^{14}$ (OWL). OWL supports the modeling of concepts and relations in such a way so that a reasoning system can make inferences upon them. For example, in our work we carefully model the spatial aspects of places within a house so that the system can later reason upon these relationships. The ability for the system to infer that "if room $\mathrm{X}$ beside room $\mathrm{Y}$ and room $\mathrm{Y}$ beside room $Z$ then room $X$ is near room $Z$ " is valuable in many health situations; for example, suggesting a patient visit their bathroom may be preceded by some system reasoning to determine if the user is "near" the bathroom in the first place. In such a system spatial relations "beside", "near", and others, are very important. Our work also allows the canonical description of common tasks - such as "take out the garbage" - which are broken down into individual steps and correlated to locations in the house. In all, the system has the potential to improve smart-homes, improve reporting, and to provide patients with highly relevant wellness and memory-related reminders, assistance, and encouragements.

Geocaching has been around for quite awhile. The ever increasing penetration of Global Positioning System (GPS) chipsets into mass-market mobile devices only increases the availability and fun of this kind of game. Geocaching is, "..a high-tech treasure hunting game played throughout the world by adventure seekers equipped with GPS devices. The basic idea is to locate hidden containers, called geocaches, outdoors and then share your experiences online.

\footnotetext{
13 The number of Americans suffering post-traumatic stress disorder is large, particularly in the community of returning war vets. Symptoms including forgetfulness and confusion are common in these individuals.

${ }^{14} \mathrm{http}: / / \mathrm{www} . \mathrm{w} 3.0 \mathrm{gg} / \mathrm{TR} / \mathrm{owl}-\mathrm{features}$
} 
Geocaching is enjoyed by people from all age groups, with a strong sense of community and support for the environment." 15 Geocaching and other sorts of augmented-reality games (in which mobile users, the physical world, and the virtual world all mesh together in a game play scenario) actually have great potential in mobile healthcare, namely because they are fun and because they encourage positive behaviors (i.e. activity, exercise). Like online multiplayer games, they have a built-in reward system in which points and reputation are sometimes achieved for finishing missions (e.g., finding geocaches, etc.). Figure 6 illustrates a geocaching application for iPhone by GroundSpeak $\operatorname{Inc}^{16}$. In the figure a player uses the application to discover the geocaches nearby her current location (the next steps would include the player walking or driving to the selected cache location, and so on.) Geocaching is a compelling mobile application, made possible by the availability of communications devices. Many feel that this is a growth application; more specifically, it has also been the subject of consideration by physical educators [22].

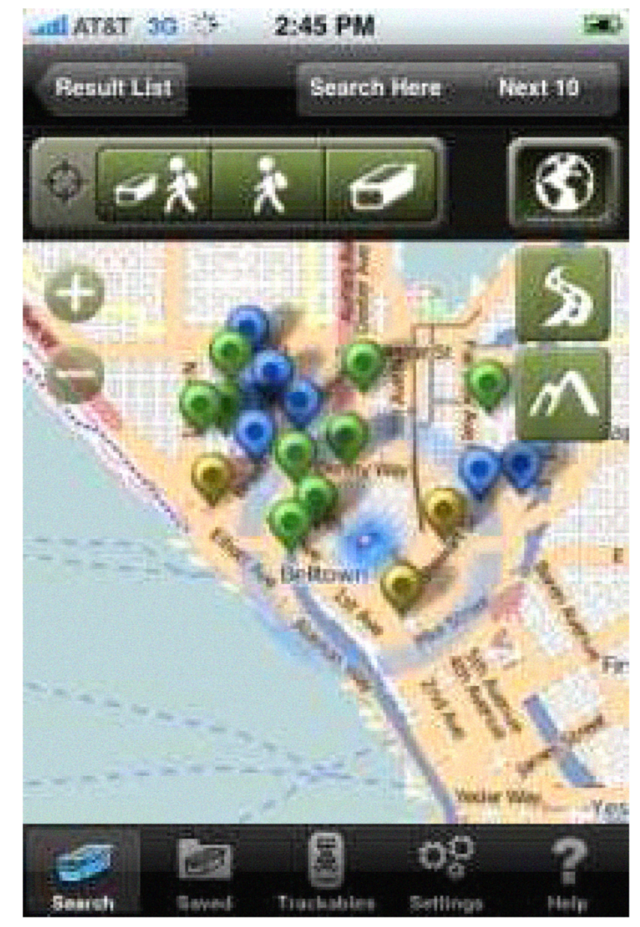

Figure 6. Outdoor geocaching application for iPhone (by GroundSpeak Inc.)

In our own prototype lab we have conceptualized a prototype called "Large Scale GPS Art" (LSGA) that encourages physical movement as well as social networking [8]. Like Geocaching, LSGA is enabled by the proliferation of devices with GPS capabilities and the programmability of device applications. Also like Geocaching, LSGA can deliver rewards and positive reinforcements which, like a feedback loop, encourage further participation and improved health. "GPS art" is the combined geo-traces of many GPS-enabled devices upon the earth (technically, air travel and sea travel can also be traced). A "geo-trace" is a series of time-stamped latitude/longitude points

\footnotetext{
${ }^{15} \mathrm{http}: / /$ www.geocaching.com

${ }^{16} \mathrm{http}: / /$ www.groundspeak.com
}

corresponding to a user's precise location. Minimally, in order to manage the interleaving and overlaying of multiple geo-traces, the traces are loosely coordinated such that they each comprise a (possibly unique) part of an image when viewed at a large enough scale. Traces might alternatively be tightly managed and assigned so as to assure that all contours of the art are covered. When viewed from a distance or at a large scale (on a digital map), the traces form the contours of the image which, in turn, can be a graphical image or logo, text, or anything else that lends itself to "contour" representation and is of interest to participants. For the group of participating users, the fun comes from taking part in a trace of a chosen image, seeing its progress over time, being able to identify (with details) which participants created which parts of the image, and the social networking and messaging opportunities.

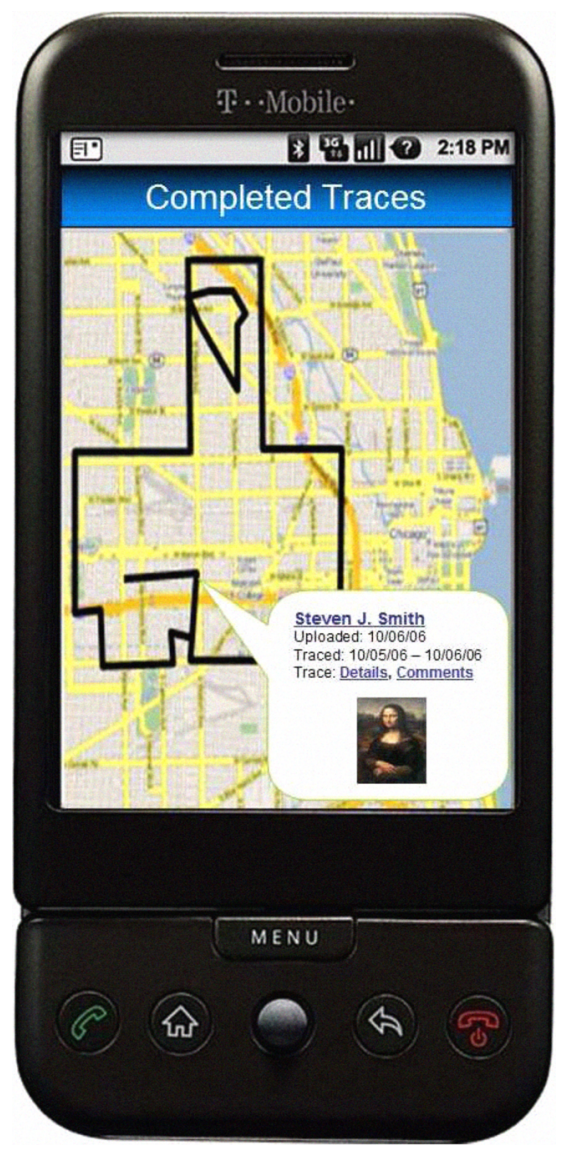

Figure 7. Geo-tracing application as a collaborative and fun endeavor [8] (emulated on an Android mobile smartphone)

Figure 7 illustrates our LSGA prototype as it would be experienced on an Android mobile smartphone. Users could alternately plan, execute, or view their current artistic "traces", or collaborate on how to achieve them in a joint fashion. In the figure a user ("Steven") examines a trace he participated in (the Chicago area) that was designed to look like the Mona Lisa painting. Once defined, this user was tasked with tracing out a part of it outline on foot or bicycle; as he does so, GPS events and logs are managed by the application and offer "proof" that this user has covered the ground necessary to draw his parts of the art. Of course the 
larger the scale of the image the more travelling and motion would be required - and the more "rewards" or health benefits might be incurred. This application can easily be adapted to smaller areas such as buildings and parks (down to the massmarket resolution of GPS, which is about a few meters, but dependent on many factors.) It could therefore be imagined that such an application may be part of a rehabilitation program or may help forgetful people stay on traces of relevant to their safety. In summary, our innovative GPS Art prototype, somewhat reminiscent of geo-caching, has a fairly clear applicability to wellness and hits a trio of requirements: 1) is fun, 2) promotes and rewards physical activity, 3) has add-on benefits related to social interactions and sense of accomplishment.

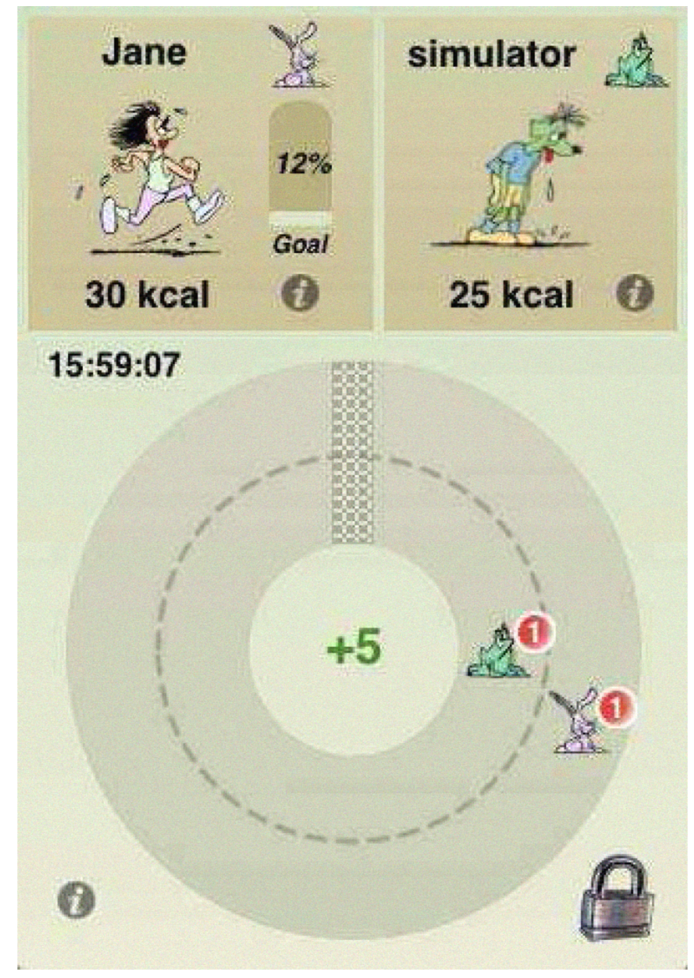

Figure 8. Walk'n'Play is a free iPhone/iPod application developed by the Univ. of Houston's Computational Physiology Lab

Walk n'Play (Figure 8) is an exemplary application for iPhone, embodying how fun challenges, competition, and whimsical design are used to encourage exercise goals [25]. Walk n'Play works in coordination with a Bluetooth pedometer, which the "player" must wear. Once a player's profile is setup (e.g., weight, age) the application tracks the players steps, considers the players weight, and computes a virtual oxygen consumption index. This index is summed during the day and the screen of the application is constantly updated. Making it more 'addictive' is the notion of competition. The player can have a competition between a friend with the same application (e.g., to see who can get most points) or with a simulated friend (see Figure 8) within the application.

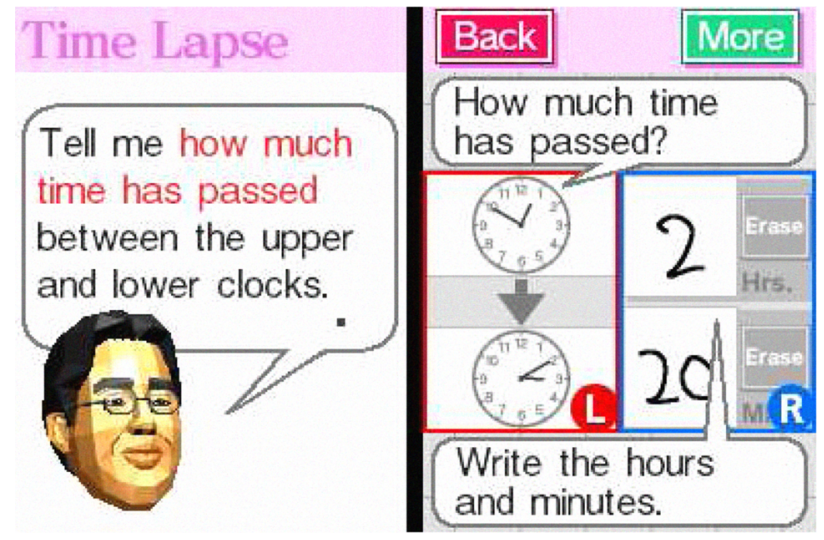

Figure 9. Brain Age by Nintendo puts users through a series of brainteasers in a fun way.

Mobile "brain games" are a relatively new trend enabled by handheld gaming platforms such as Nintendo DS and (more recently) smartphones. There is not consensus about which specific types of brain activities may be best but there is wide consensus that continual learning into the elderly years can push back the onset of cognitive diseases such as Alzheimer's. Designers have made brain games a fun and motivational new fad, building on the mass-market interest in trivia and problem solving (e.g., Sudoku). Brain games are generally fast-paced and use simple clear graphics. As they need to appeal to a wide gamer-base (from young to old), "keeping it simple" is a key design requirement with games guiding users through a series of tasks, congratulating and rewarding and encouraging further game play. Figure 9 shows a Nintendo brain game while Figure 10 shows one by Glu Games Inc.

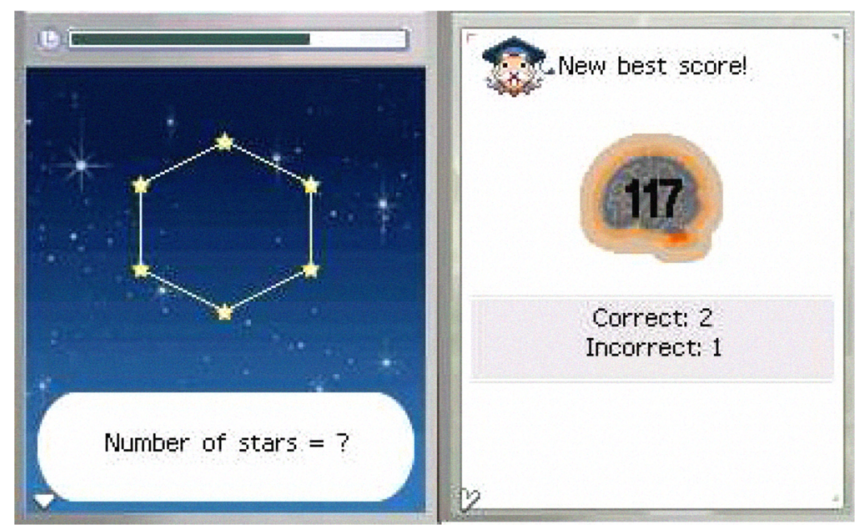

Figure 10. Brain Genius by Glu Games Inc.- two screens shown: a challenge (left) and a summary/reward (right)

In summary, a major functional theme of mobile healthcare will be that of encouraging and mitigating patient activity. From a designer's point of view, this means building attractive and compelling interfaces that are able to nudge users towards activity via some motivational impetus. This may involve, for example, designing in rewards and positive re-enforcements in a clever way, making activities so fun and engaging that patients "forget" that they are active (e.g., Wii, Brain Games, Geocaching, etc.), or enabling a social communications with positive role models and mentors [2]. 
DESIGNING REPRESENTATIONS OF MEDICAL CONTEXT AND REPORTS

This section outlines several interesting and representative interface techniques that allow medical and health context and information to be better appreciated or utilized. Representation and analysis of data is a key requirement of many mobile healthcare use cases in: telemedicine, remote monitoring, remote consultation, and consumer-facing wellness applications. Consumer applications are often required to display biometric sensor readings (e.g., in conjunction with consumer Bluetooth heart rate and blood pressure monitors such as those from BodyTel Inc.) in highly readable fashion.

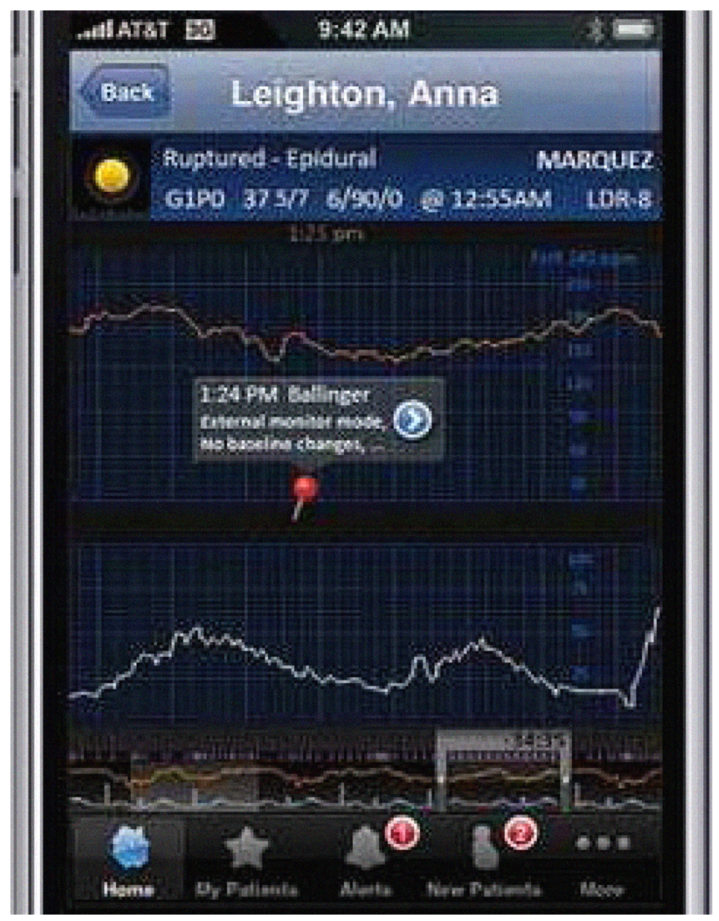

Figure 11. Intrapartum live waveform by Airstrip

Graphical smartphone mobile applications for platforms such as Windows Mobile and iPhone are slowly gaining FDA approval for use in hospital settings. A representative example is AirStrip Technologies ${ }^{17}$ whose mobile healthcare applications are used in Labour and Delivery (L\&D) units. At its core, AirStrip "facilitates the transfer of real-time, medical waveform ${ }^{1}$ data to a Cell Phone (Smartphone) or PDA over a cell phone network," and focuses on security reliable transmissions. Figure 11 illustrates the kind of crisp and highly usable charting featured in the Intrapartum application.

AirStrips healthcare applications are binary applications for mobile OS's. Many of today's mobile services, in fact, are Web-based services and use Web technologies to build them: HTML, Javascript, AJAX, Flash, etc. iPhone applications are built with Cocoa, while Android and MIDP phone apps are built using Java. Palm Pre applications will highly leverage technologies of the Web for the mobile platform: Javascript, xml, etc. Drupal and Google Charts API are examples of

${ }^{17}$ www.airstriptech.com graphics building blocks. The former is Flash API for creating charts in Web apps from dynamic data while the latter is a Javascript API for charts; both are capable of diverse chart types including: pie, bar, 3D, scatter, area, and so on. Figure 12 illustrates how with these building blocks, developers need be concerned only with passing data to the charting API's rather than in implementing charting logic.
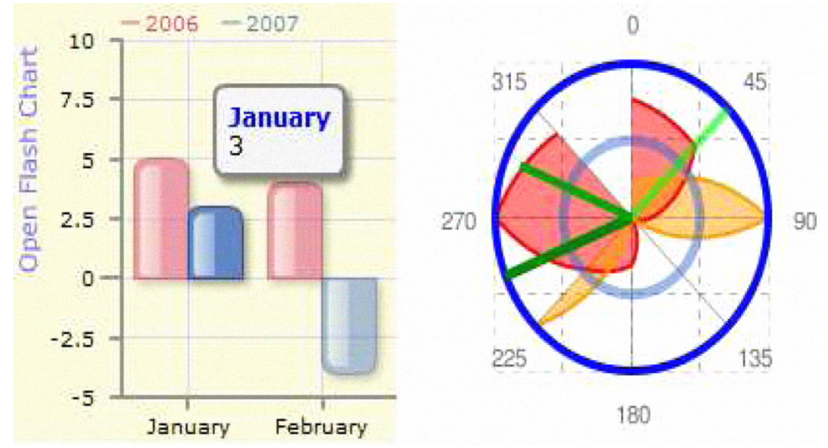

Figure 12. Charting is a key requirement of many consumer and clinician mobile healthcare applications; Drupal (left) and Google Charts API (right)

Mobile Tiles [7] (Figure 13) is an advanced mobile application intended to enable non-linear browsing of temporal media - such as biomedical sensor data, charts, radiology imagery, etc. - in a light-weight pixel-efficient manner on mobile smartphones. Tiles employs a unique and effective interactive visual layout in which media segments pulled from a remote server or local cache - are organized in multiple "perimeter rings" around a central image (see Figure 13).

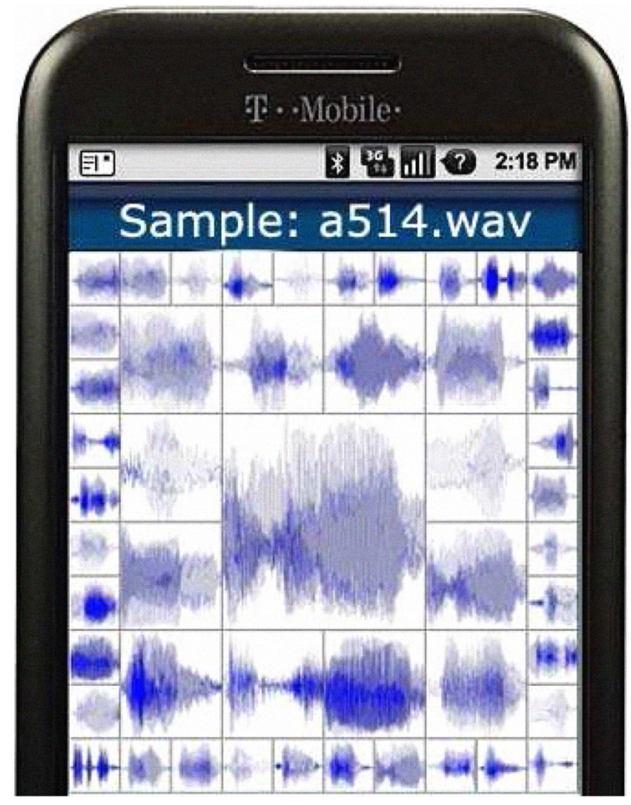

Figure 13. (partially emulated) Mobile Tiles application of waveform analysis and browsing

The central image corresponds to a "focus" while the images on the rings are sampled from the current temporal "range". This paradigm gives the user a flexible way to quickly view detail as well as context important to analysis. Keypad and 
screen taps on individual tiles in the rendering shift the current focus to the tapped segment and cause a redraw of the range segments surrounding it. The temporal range of view can be changed be using zoom-in and zoom-out taps or buttons. Tiles is a surprising, fun, and effective way to browse any visual data that can be ordered on a parameter, such as time, or place. The figures illustrates Tiles in a scientific scenario; in this figure an analyst is using the tool to remotely browse clinical data such as audio waveforms (in the figure a long waveform is broken into segments corresponding to words in a oratory), heart-rate streams, or blood pressure readings. At each viewing interaction, the analyst may decide to either "play" one of the segments displayed, "zoom in" to more detail in the stream, or refocus the representation on another area of the stream. Tiles currently executes on a Java MIDP platform and is being ported to others platforms and media and health applications.

Life logs, or life blogs are the collected digital artifacts of a person's experiences stored in digital form. For example, the Nokia lifeblog application grouped all the photos, blogs, MMS, and SMS, of a given user into a time-lined multimedia diary (see Figure 14). The result was searchable and sharable. Any given user could view his own multimedia timeline using the mobile device; when this was requested a series of media (e.g., text, imagery) blocks were presented on the screen corresponding to the time in question. These kinds of applications are relevant to healthcare because they teach us the art of presenting diverse information on small mobile screens, allow us to store and search for personal (possible wellnessrelated) data, and are increasingly seen - in a more serious sense - as memory-aides for people with memory impediments.

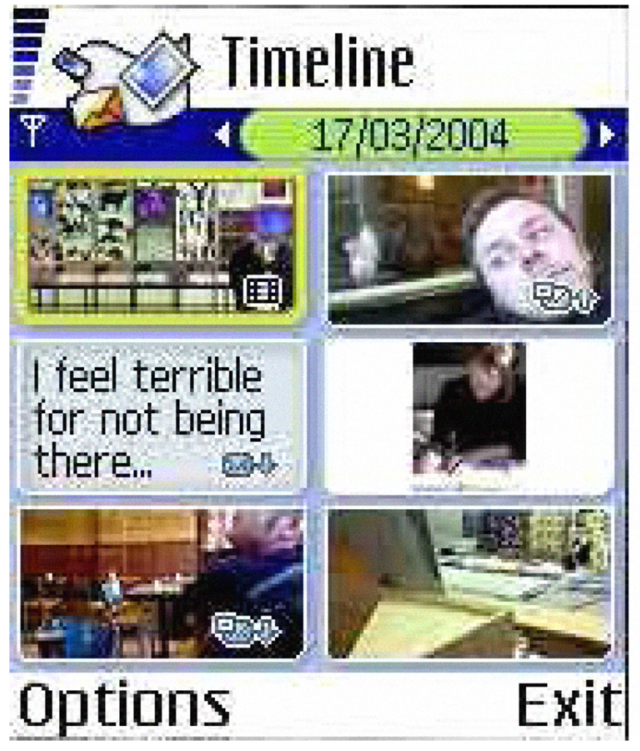

Figure 14. Nokia LifeBlog interface

The challenges of data presentation on small mobile screens become clear when examining LifeBlog. The designers have made good use of the mobile screen space, filing most of the screen with informative information while preserving a layout that is intuitively simple. Information-density can be a desirable trait of a mobile service on a small screen [12][18]; our own Tiles applications similarly uses a pixel efficient rendering.
QVGA (320x240 pixels) is roughly typical for today's smartphones.

Finally, a relatively large number of mobile applications now allow users to self-diagnose, report and log their health issues as they arise, and look up further information (of course, anyone with a data connection can use the Wikipedia as well). The application A.D.A.M. mobile ${ }^{18}$ uses a simple and clean interface designed specifically to allow users to explore symptoms by tapping on an image of the human body, which results in further detailed information. Empowering users with information in intuitive visual ways will be a key requirement in mobile healthcare applications. Figure 15 illustrates the A.D.A.M interface.

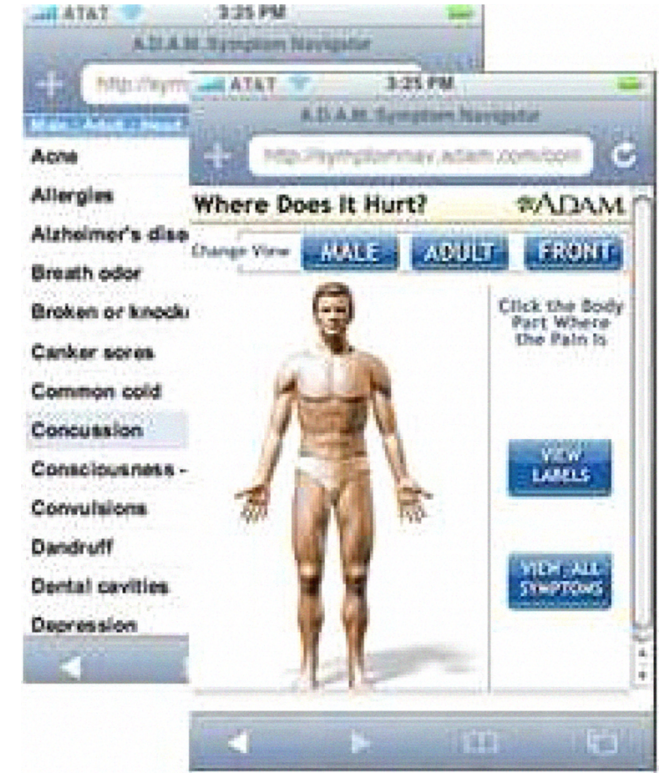

Figure 15. Exploring symptoms through an intuitive visual interface

Figure 16 illustrate a mobile application that users buy to use as a comprehensive tracker of personal datapoints. TheCarrot ${ }^{19}$ is a mobile (and Web) applications that tracks health statistics and presents them on demand in many formats, including a calendar formatted one. DiaMedic ${ }^{20}$ is a similar application aimed at diabetics. LifeScan.com is working on innovative diabetes management tools for mobile phones that will allow the remote monitoring of a child's blood levels. Epocrates ${ }^{21}$ for iPhone is a popular reference application from which users can research pill interactions (see Figure 17), formulae, drug interactions, and more. It makes good use of menus and features welldesigned clear photographs of pills to aid in identification. A subscription-based version, touted as, "Integrated drug, disease, and diagnostic suite for mobile devices" is also available in which the reference data is updated over-the-air.

In summary, the representation of medical and contextual information on mobile devices is a key part of mobile healthcare application scenarios. This is in some ways obvious and echoes the broad arcs of human computer

\footnotetext{
${ }^{18} \mathrm{http}: / /$ iphone.adam.com

${ }^{19} \mathrm{http}: / /$ thecarrot.com

${ }^{20} \mathrm{http}: / /$ www.martoon.com/diamedic/

${ }^{21} \mathrm{http}: / / \mathrm{www} . e p o c r a t e s . c o m /$ products/rx/iphone.html
} 
interaction research [19]. Mobile healthcare patients will cut across age and social divides; obese teenagers, wounded warriors, elderly patients. Simplicity and usability will be important. Social and situational context will need to be considered during design, as will using the right mix of graphics (and sounds) that neither pander to, nor overwhelm, nor confuse the patient.

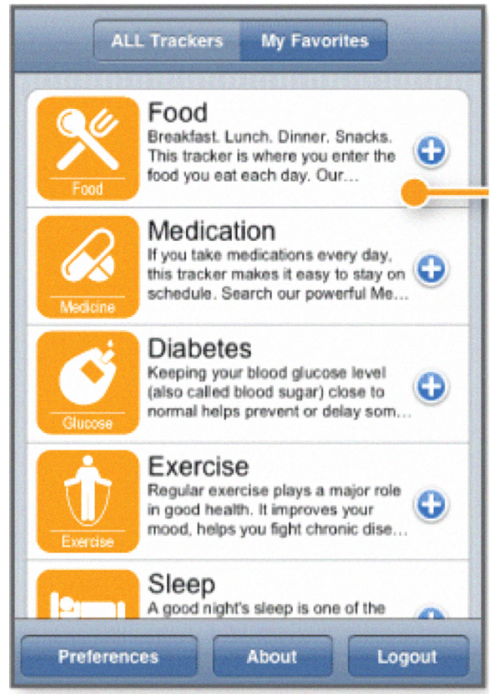

Figure 16. Using the mobile device as a tracker via TheCarrot tools

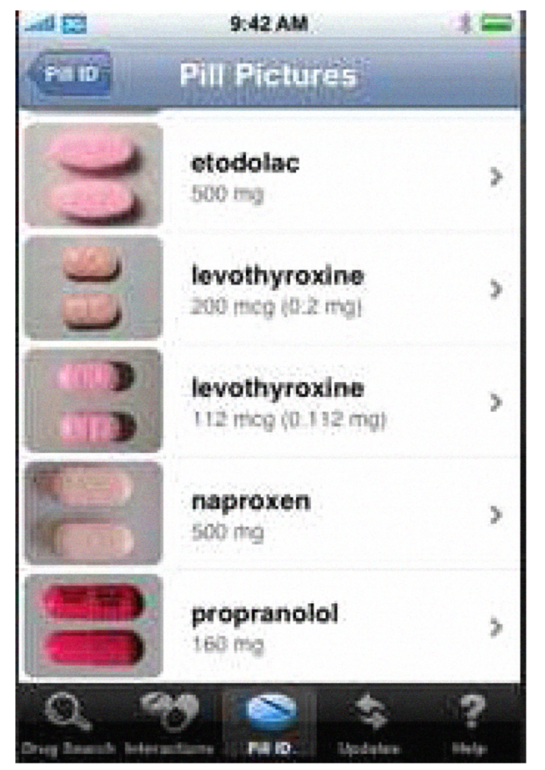

Figure 17. Epocrates reference medical application

\section{CONCLUSIONS}

This paper has presented some important examples of mobile technologies, underpinnings, and services that are key pieces of the mobile healthcare ecosystem. We have focused on relevant novel aspects of our own applied research, a literature search, as well as mass-market consumer offerings. Put together, these examples both illustrate and reinforce our argument that creative mobile applications and services will help increase the adoption of mobile healthcare.
Web 2.0, Bluetooth and Zigbee sensors, online health records, programmatic API's, and software development kits are some of the "enablers" of mobile healthcare. Microsoft Surface, iPhone/iTunes, Nintendo Wii, iPhone, Android, J2ME, and Web browsers are some of the (Internetconnected) "platforms". Together with the creativity of application developers, a new and fascinating array of mobile healthcare applications are now possible. Just as the iPhone programmability made applications like, for example, G-Park (in which users locate where they parked their car) viable, new and useful healthcare applications will emerge by leveraging the enablers. Already, there are dozens of healthcare related applications available on various mobile platforms. Many of these applications - sometimes because they are written by individuals or small startups - are modest in functionality or are highly derivative. Still others seem to have found a "sweet spot" niche, either by doing a single thing very well or by filling a cool, fun or marketable niche. Our own R\&D [3][4][5][6][7]8] exhibits several exemplary aspects that help re-enforce our themes.

For mobile designers, there is no magic formula. In this paper, however, we identified some of the broad themes in visual and interaction design that make for compelling and useful mobile healthcare applications; they are illustrated in Figure 18 and summarized below:

- Designing for a range of novel and familiar interfaces

- Designing to encourage physical and mental activity

- Designing for simple conveyance of health info

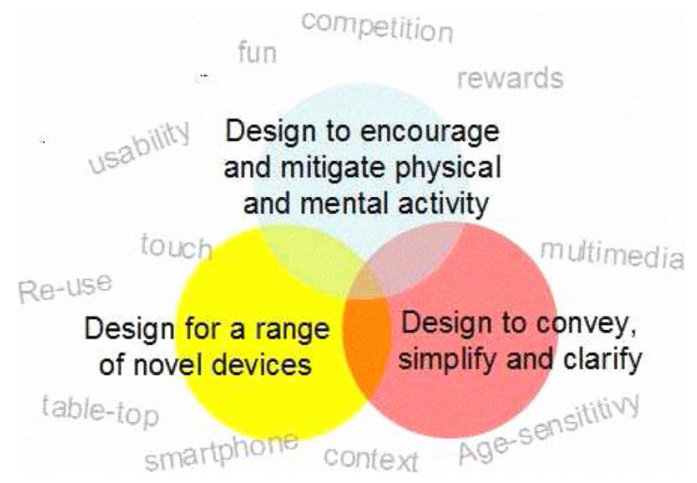

Figure 18. High level visual and interaction themes and keys

At the center of the figure, where the three themes overlap, lies the "sweet-spot" for mobile healthcare. Applications in this region have successfully considered these important themes in their design and are in a better position to succeed. This paper has surveyed several mobile healthcare applications that would lie in and around this sweet-spot.

These are exciting times for both users and developers of mobile services. The new systems and tools that make human-computer interaction easier than ever before also allow the expression of creativity on new types of media. If mobile healthcare manages to adopt the right pieces at the right time and in the right way, society will greatly benefit from improved management and delivery of medical care. While there are many hurdles at legislative (e.g., in US see FDA, HIPAA), liability, and usability levels, most are optimistic that mobile healthcare services will one day go 
beyond simplistic use cases and actually encourage us, reward us, interpret medical information for us using graphics, allow us to communicate with our care (social) network, and use pervasive and artificial intelligence technologies to provide unprecedented value.

\section{REFERENCES}

[1] S.Loeb, B.Falchuk, E.Panagos, The Fabric of Mobile Services, John Wiley \& Sons, New York, 2009

[2] N.Christakis, J.Fowler, "The Spread of Obesity in a Large Social Network over 32 Years", New England Journal of Medicine, Volume 357:370-379

[3] B.Falchuk, A.Misra, S.Loeb, "Server-assisted Context-Dependent Pervasive Wellness Monitoring", Proc. ICST International Workshop on Wireless Pervasive Healthcare, London, 2009

[4] B.Falchuk, K.Sinkar, A.Dutta, S.Loeb, "Mobile Contextual Mashup Service for IMS", Proc. $2^{\text {nd }}$ IEEE Int'l. Conf. on Internet Multimedia Services Arch. and Applications, Bangalore, Dec. 2008

[5] B.Falchuk, S.Loeb, "Towards Guardian Angels and Improved Mobile User Experience", Proc. IEEE GLOBECOM 2008 Communications Software \& Services Symp., New Orleans, 2008

[6] B.Falchuk, E.Panagos, S.Loeb, “A Deep-Context Personal Navigation System", Proc. ITS America $15^{\text {th }}$ World Congress on Intelligent Transportation Systems, New York, 2008

[7] B.Falchuk, A.Glasman, K.Glasman, "Tool for Video Content Understanding on Smartphones", Proc. $10^{\text {th }}$ ACM Int'l. Conf. on Human-Computer Interaction with Mobile Devices \& Services, Amsterdam, 2008

[8] B.Falchuk, "Web Application Supporting Large-Scale, Collaborative, Geo-traced Art", Proc. IASTED Int'l. Conference on Web Technologies, Applications, and Services (WTAS'06), Calgary, 2006

[9] S.Almasy, "Invention turns cell phone into mobile medical lab", available at: http://www.cnn.com/2009/TECH/02/03/ medical.imaging.device/index.html

[10] S. Yang, "Engineers Harness Cell Phone Technology for Use in Medical Imaging." UC Berkeley Science Daily, May 1, 2008.

[11] American Heart Association Report, "Excess weight, inactivity increase heart failure risk in men", available at: http://www.americanheart.org

[12] Studio 7.5, "designing for small screens", Ava Publishing, Switzerland, 2005

[13] C.Phend, "Teen TV Habit Leads to Adult Dietary Woes", Medpage Today, http://www.medpagetoday.com/PrimaryCare/DietNutrition/12672

[14] C.Leto, "Seniors say 'Wii",, Senior Advocate, http://www. senioradvocatenews.com/

[15] S.Consolvo et al, "Design Requirements for Technologies that Encourage Physical Activity", Proc. ACM CHI'06, Montreal, 2006

[16] G. Bond, L.S. Aiken, S.C. Somerville, "The health belief model and adolescents with insulin-dependent diabetes mellitus", Health Psychology, Vol 11(3), pp.190-198, 1992

[17] S.Davidoff et al, "Principles of Smart Home Control", Proc. UbiComp '06: Ubiquitous Computing, Orange County California, 2006

[18] E.Tufte, Envisioning Information, Graphics Press, 1990

[19] K.Siau, Z.Shen, "Mobile healthcare informatics", Informatics for Health and Social Care, 31(2), Taylor and Francis, 2006

[20] H.Knight, J.Lee, H. Ma, "Chair Alarm for patient fall prevention based on Gesture Recognition and Interactivity", Proc. IEEE Int'l Conf. on Engineering in Medicine and Biology Society, 2008
[21] F. Sainz Salces, M. Baskett, D. Llewellyn-Jones, D. England, "Ambient Interfaces for Elderly People at Home", in Ambient Intelligence in Everyday Life, Springer, 2006

[22] B. Schlatter, A.Hurd, "Geocaching: 21st-Century Hide-andSeek", Journal of Physical Education, Recreation \& Dance (JOPERD), 76(7), pp.28, Sept. 2005

[23] S.Mason, R Bohringer, J Borisoff, G Birch, "Real-Time Control of a Video Game with a Direct Brain-Computer Interface", Journal of Clinical Neurophysiology. 21(6):404-408, November/December 2004

[24] M.Pollack, "Intelligent Technology for an Aging Population", AI Magazine, 26(2), pp.9-24, 2005

[25] L.Merkl, "Every Move You Make: Free Smart Phone App Helps Burn Calories", Univ of Houston newsletter, http://www.uh.edu/news-events/archive.php, May 2009

Ben Falchuk obtained a Bachelor of applied mathematics and computer science from University of Waterloo (1993), a Master of science from Carleton University (1995), and a Ph.d. in electrical and computer engineering from University of Ottawa (1999). He is currently a Senior Research Scientist at Telcordia Technologies in Piscataway, NJ, USA and his research areas include mobile applications, human-computer interaction, interactivity, and communications middleware and services. Dr. Falchuk is a member of the ACM and the IEEE Computer Society, is a journal editor-inchief, and serves on international program committees and editorial boards of conferences and journals. 